\title{
Glutamate receptor ion channels
}

\author{
Mark L Mayer
}

Glutamate receptor ion channels mediate excitatory responses at the majority of CNS synapses. They are the only ligand gated ion channels for which multiple high resolution crystal structures have been solved. Highlights of information gained from mechanistic studies based on crystal structures of their ligand binding domains include explanations for strikingly diverse phenomena. These include the basis for subtype specific agonist selectivity; mechanisms for desensitization and allosteric modulation; and mechanisms for partial agonist activity. In addition, multiple lines of evidence, including low resolution electron microscopic studies, suggest that native AMPA receptors combine with an auxiliary subunit which regulates activity and trafficking. Functional studies suggest that glutamate receptor gating is distinct from that of structurally related voltage gated ion channels.

\section{Addresses}

Laboratory of Cellular and Molecular Neurophysiology, Porter Neuroscience Research Center, Building 35 Room 3B 1002 MSC 3712, 35 Lincoln Drive, Bethesda MD, 20892 3712, USA

\author{
Abbreviations \\ ACBC 1-aminocyclobutanecarboxylic acid \\ ACPC 1-aminocyclopropanecarboxylic acid \\ AMPA $\alpha$-amino-3-hydroxy-5-methyl-4-isoxazole propionic acid \\ ATPO (S)-2-amino-3-[5-tert-butyl-3-(phosphonomethoxy)-4-isoxazolyl]propionic acid \\ iGluR glutamate receptor ion channel \\ DNQX 6,7-Dinitro-2,3-quinoxalinedione \\ NMDA N-methyl-D-aspartic acid \\ TARP transmembrane AMPA receptor regulatory protein
}

\section{Introduction}

Ligand gated ion channels are membrane proteins which mediate information transfer at synapses. Their function relies on the ability to respond very rapidly to the transient release of a neurotransmitter to produce a change in membrane potential of the postsynaptic cell. Genome and cDNA sequencing analysis reveals that there are three major subtypes of neurotransmitter activated ligand-gated ion channel, each with a unique architecture. These are the glutamate receptors, tetrameric cation selective channels which are activated by glutamate and a number of environmentally significant plant neurotoxins; the so called cys-loop receptors, which form pentameric ion channels gated by acetylcholine, GABA, glycine and serotonin; and the $\mathrm{P} 2 \mathrm{X}$ receptors which are believed to be trimeric ion channels gated by ATP.

The glutamate receptor ion channels (iGluRs) are abundantly expressed in the brain and spinal cord and mediate responses at the vast majority of excitatory synapses. 
Mammalian iGluRs are encoded by 18 genes which assemble to form four major families, the AMPA, kainate, NMDA and delta receptors. There are four AMPA receptor genes (GluR1-4); five kainate receptor genes (GluR5-7, plus KA1 and KA2); seven NMDA receptor genes (NR1, NR2A-D, NR3A and NR3B); and two delta subunits. Coassembly of iGluRs within but not between families, generates a large number of receptor subtypes in vivo. Unique amongst ligand gated ion channels, high resolution crystal structures have been obtained for multiple subtypes of iGluRs, and such structures provide the means to gain unprecedented insight into their mechanism of action and modulation. The unique architecture of iGluRs is a key factor which has facilitated their structural analysis (Figure 1). Unlike the cys-loop receptors, in which ligand binding sites are formed at the interface between subunits, in iGluRs the ligand binding cores are discrete domains, one copy of which is present in each subunit. The ligand binding domains can be genetically excised, expressed as water soluble proteins, and crystallized. To date structural analysis has been successfully applied to the ligand binding cores of AMPA [1], kainate [2] and NMDA [3] receptors; work is ongoing in several labs on other iGluR domains but the experience gained indicates that these are more challenging targets. Due to this difficulty in solving structures for the amino terminal, and ion channel domains, significant new information continues to emerge from more traditional cell biological and genetic based approaches. The material covered in this review focuses on experiments designed to resolve the molecular mechanisms of iGluR gating and does not address the topics of targeting and synaptic function.

\section{Agonists antagonists and ligand binding domains}

Prior structural work on AMPA receptors established that the ligand binding cores assemble as pairs of dimers, and suggested that agonist stabilized domain closure of the individual subunits in a dimeric assembly leads to channel opening. Graded closure of the ligand binding cores produced partial agonist responses. Desensitization resulted from a reorganization of the dimer assembly which allowed the channel to close even though the individual ligand binding cores remained in their active agonist bound conformation. A substantial body of new experimental work reinforces this picture. Crystallization of the ligand binding domains of the kainate receptor GluR5 [2,4] and GluR6 [2,5] subunits, in conjunction with previously solved structures for the glycine binding NMDA receptor NR1 subunit [3] and the AMPA receptor GluR2 subunit [6], permits for the $1^{\text {st }}$ time an understanding of the molecular mechanisms underlying the subtype specific ligand selectivity characteristics of individual iGluR subunits. The overall fold of the iGluR ligand binding domains for AMPA, kainate and NMDA receptors is nearly identical. For GluR6 expression in insect cells provides for the $1^{\text {st }}$ time information about the structure of glycosylation sites [2,5]. Key amino acid side chains which interact with the ligand $\alpha$ $\mathrm{NH} 2$ and $\alpha-\mathrm{COOH}$ groups are the same in all four iGluR families. What differs are the amino acids which interact with the glutamate $\gamma-\mathrm{COOH}$ group or in the case of NR1 subunits prevent the binding of glutamate. In the agonist bound complex of all iGluRs the ligand is buried in the interior of the protein, but the volume of the ligand binding cavity varies substantially, from $56 \AA^{3}$ for NR1 to 218,305 and $255 \AA^{3}$ for GluR2, GluR5 and GluR6, respectively (Figure 2). One amino acid in the NR1 subunit glycine binding pocket, a Trp side chain at position 731, plays a key role in selectivity by occupying space required for the glutamate $\gamma-\mathrm{COOH}$ group; as a result glutamate cannot bind to the 
NR1 subunit [3]. In GluR5 and GluR6 steric occlusion mediated by Ser/Asn and Leu/Phe exchanges play key roles in subtype selectivity [2,5]. Strikingly, in the NR1 binding pocket there is no ordered solvent, while in the GluR2, GluR5 and GluR6 glutamate complexes there are 4, 6 and 5 bound water molecules. The displacement of solvent permits the binding of large heterocyclic agonists, such as quisqualate, ATPA and AMPA, with relatively little or in some cases no change in the extent of domain closure $[2,6,7]$. Notably the larger volumes of the kainate receptor ligand binding cores do not result from differences in the extent of domain closure, since for both GluR5 and GluR6 the ligand binding cores are more closed than for GluR2. NMR measurements for AMPA receptor binding cores are in their early stages but reveal subtle differences in conformational flexibility for individual agonist complexes [8] and line broadening for residues in the dimer interface [9].

Substantially less work has been done with iGluR antagonist complexes. The structure of a GluR2 complex with ATPO reveals the same open cleft conformation but a different set of ligand protein interactions than observed previously for the quinoxaline DNQX; of note the phosphonate group in ATPO acts as a substitute for a $\left[\mathrm{SO}_{4}{ }^{2-}\right]$ ion observed in the DNQX complex [10]. For kainate receptors no antagonist structures have been solved but a molecular model has been developed for a novel GluR5 selective antagonist [11]. For the NR1 subunit of NMDA receptors structures of complexes with 5,7,dichlorokynurenic acid and cycloleucine also reveal the now familiar open cleft conformation for competitive antagonists $[3,12]$. Of potential significance for functional studies on receptor turnover and trafficking in vivo is development of a photoactive irreversible AMPA receptor antagonist [13]. Also of note was the development of thiol reactive NR1 antagonists used for modeling the structure of the NR1 ligand binding core [14]. To date structures for NMDA receptor NR2 subunit glutamate binding domains have not been reported but mutagenesis and molecular modeling suggests plausible models $[15,16]$.

\section{Mechanisms for partial agonist action}

Partial agonists, compounds which produce less than the maximum response of full agonists, are powerful tools with which to probe receptor gating mechanisms.

Historically, two distinct models have been proposed to explain such behavior. The MWC concerted transition model, in which receptors exist in an equilibrium between resting and active conformations, explains difference in agonist efficacy as resulting from different relative affinities of agonists for the resting and active states. The induced fit model suggests instead that the receptor can exist in multiple conformational states and that the ligands which best fit the receptor binding pocket produce the largest extent of

activation. Crystal structures for the ligand binding cores of wild type and mutant AMPA, kainate and NMDA receptor complexes indicate that both of these strikingly different mechanisms underlie partial agonist activity. In the most extensive analysis performed a series of 5-substituted willardiines was shown to promote graded closure of the AMPA receptor ligand binding core consistent with the induced fit model [17]. Variation in the extent of domain closure due to steric occlusion, which was correlated with the efficacy for activation of channel gating, and inversely with the extent of desensitization, strongly suggests an induced fit model. Similar observations on individual compounds including 
kainate, a very weak partial agonist [18], (S)-2-amino-3-(4-bromo-3-hydroxy-isoxazol-5yl)propionic acid [19] and CPW399 [20] stronger partial agonists, reinforce this model. Surprisingly, a similar analysis of the AMPA receptor L650T mutant, reveals a second mechanism for partial agonist action at AMPA receptors, reduced stability of the fully closed agonist bound conformation as required by the MWC model [18]; notably this occurred only for AMPA which produced $38 \%$ of the maximum response, with glutamate and quisqualate continuing to act as full agonists. Spectroscopic studies on the related GluR4 L651V mutant provide another biophysical approach to measure ligand induced conformation changes [21]. For the NR1 subunit of NMDA receptors the partial agonists D-cycloserine, ACPC and ACBC all produce the same extent of domain closure as the full agonists glycine and D-serine again consistent with the MWC model $[3,12]$. For kainate receptors less work has been done, and notably the structure of the Apo state or an antagonist bound complex has yet to be solved to provide a reference point for comparison with other iGluR subtypes. For GluR6 the kainate and domoate complexes are 3 and 14 degrees more open than the glutamate complex, consistent with the action of kainate as a partial agonist of much higher efficacy for GluR6 versus GluR2 and an induced fit mechanism for partial agonist action based on domain closure [2,5,22].

\section{Ligand binding core dimer assemblies}

Different from the cys-loop receptors the ligand binding cores of iGluRs are believed to assemble as a dimer of dimers. The dimeric assembly of iGluRs was first described for AMPA receptors and is generated by apposition of the backside of the domain 1 surfaces of each subunit, burying a solvent accessible surface of about $2400 \AA^{2}$ [6]. Stabilization of dimer contacts at the domain 1 interface is known to attenuate AMPA receptor desensitization [24]. Similar dimer crystal structures have now been observed for the ligand binding cores of the NR1 [3] and GluR6 subunits [5] and at low resolution in single particle electron microscopic images of intact AMPA receptors [23]. Although the $3.1 \AA$ resolution of the GluR6 crystal structure is too low to reveal details of the dimer interface, it appears that many of the contacts present in AMPA receptors are conserved. Surprisingly, a strikingly different dimer structure was observed in the GluR5 glutamate complex [5]. In the GluR5 dimer the pair of subunits have separated at their domain 1 interface; because this is accompanied by a rotation of each subunit the separation of the linkers leading to the ion channel segments remains unchanged from that in the AMPA like dimer observed for GluR6. As a result the novel packing observed in the GluR5 dimer is unlikely to correspond to the desensitized state and is more likely a consequence of crystal packing forces.

\section{Desensitization}

The rapid and profound desensitization which follows activation of iGluRs has provided a powerful experimental tool for analysis of the underlying allosteric mechanism. Prior crystallographic, biochemical and functional studies for GluR2 revealed that domain 1 ligand binding core dimer contacts must be broken for desensitization to proceed [24]. Extensive tests using site specific mutagenesis to disrupt domain 1 intermolecular contacts observed in AMPA receptor dimers strongly reinforce the conclusion that the dimeric assembly observed in crystal structures of the isolated ligand binding cores is present in full length membrane bound AMPA receptor ion channels [25]. Single particle 
electron microscopic analysis of intact AMPA receptors purified from rat brain reveals that this mechanism now has to be extended to include movement of the associated $\mathrm{N}$ terminal domains [26]. Surprisingly, despite $88 \%$ amino acid sequence identity in the ligand binding cores of AMPA and kainate receptors, mutations in the GluR6 dimer interface which would be expected to perturb intersubunit contacts and accelerate desensitization have the opposite effect [22]. However, only a limited set of mutants was studied, and glycine instead of alanine was used to replace GluR6 side chains, potentially allowing the main chain to adopt conformations inaccessible to other amino acids due to removal of steric constrains. Therefore, additional experiments are required to evaluate potential differences in AMPA and kainate receptor gating. A notable feature of the established model for AMPA receptor desensitization is that it defines the active state in great detail but reveals nothing about the structure of the desensitized state itself the crystal structure of which has not been determined. The report that mutations in the linker between the $2^{\text {nd }}$ transmembrane segment and the ligand binding core disrupt desensitization of both AMPA and kainate receptors, albeit to different extents, provides the $1^{\text {st }}$ clues about conformational states that are occupied during desensitization [27]. Analysis of the action of novel allosteric modulators of AMPA receptor desensitization confirms a key role for the dimer interface and extends the binding site beyond residues known to interact with the drug cyclothiazide $[28,29]$.

\section{Auxiliary subunits}

iGluRs coassemble with a vast horde of cytoplasmic and cytoskeletal proteins via PDZ binding motifs and numerous other signals [30]. A growing body of evidence indicates that these proteins modulate iGluR function. For example, coexpression of GluR6 and PSD 95 accelerates the rate of recovery from desensitization 2-fold [31]. However, none of these proteins can be considered true accessory subunits as found for example in voltage gated ion channels. This situation has now changed as a result of recent studies which show convincingly that a subset of membrane proteins, that are members of a gene family which includes calcium channel $\gamma$ subunits, interact specifically with AMPA receptors to modify both their transport to the plasma membrane and their gating properties $[32,33,48]$. These are named transmembrane AMPA receptor regulatory proteins (TARPs). Native PAGE, single particle electron microscopy, mass spectral analysis of affinity purified native AMPA receptors from rat brain, which were decorated with Fab fragments, independently established the coassembly of AMPA receptors with TARPs, and revealed substantial density in the transmembrane region that was attributed to TARPs $[26,34]$. Amongst many notable aspects in these studies is the observation that the kinetics of AMPA receptor and synaptic responses are modified by TARPS; this is noteworthy because it raises the prospect that there may be accessory proteins for other iGluR subtypes, especially kainate receptors, for which there is a striking mismatch between the kinetics of synaptic responses and those of recombinant kainate receptors in heterologous expression systems.

\section{Modulation assembly and gating}

NMDA receptors are notable for many characteristic properties, including complex kinetics, allosteric modulation by ligands acting at the amino terminal domain, and the fact that they are obligate heteromers. The description of modal gating adds to this 
complexity [35]. Detailed kinetic analysis permits for the $1^{\text {st }}$ time a physical model of proton inhibition [36] and a description of NMDA receptor gating during repetitive synaptic activity [37]. Analysis of NMDA receptors formed by coexpression of the NR1, NR2A and either NR2B or NR2C subunits reveals that the inhibitory action of nM Zn ${ }^{2+}$ at the NR2A subunit persists in triheteromeric assemblies which likely form NMDA receptor complexes in vivo, but that the inhibition saturates at about 15\% [38]. The much lower affinity inhibition of the NR2B subunit by $\mathrm{Zn}^{2+}$ has the consequence that in native NMDA receptor assemblies containing both NR2A and NR2B subunits $\mathrm{Zn}^{2+}$ modulation occurs over a broad concentration range and may have both tonic components mediated by $\mathrm{nM} \mathrm{Zn}^{2+}$ as well as an activity dependent component mediated by vesicular release of $\mathrm{Zn}^{2+}$ [39]. Similar to NMDA receptors it has now been established that kainate receptors show modulation by extracellular protons and polyamines [40] while on the other hand cytoplasmic polyamines, long established as modulators of AMPA and kainate receptors also modulate NMDA receptors [41]. The mechanisms underlying modulation of GluR5 subtype kainate receptors by endogenous ligands is less well understood than for NMDA receptors, in part due to difficulties in achieving sufficient levels of expression for routine functional analysis. The discovery that the GluR5b splice variant has uniform kinetic properties compared to the 5a splice variant, and that mutations in the C-terminus greatly increase the cell surface expression of GluR5b, opens up the hope for more detailed functional studies [42].

Regulation of the assembly and trafficking of iGluRs is beyond the scope of this review but a couple of interesting themes are relevant to iGluR function. Surprisingly it is possible to express functional NMDA receptor tandem constructs in which NR1 and NR2 subunits truncated after the $2^{\text {nd }}$ transmembrane segment were fused to wild type subunits and coexpressed with the missing final TM and intracellular $C$ terminus [43]. Another unexpected result was the demonstration that agonist binding is a critical determinant for plasma membrane expression of kainate receptors $[44,45]$. The underlying mechanisms have yet to be determined as has the ligand occupancy of iGluRs in the endoplasmic reticulum, golgi apparatus, and other intracellular membrane bound environments. Also of note is the observation that the assembly of AMPA receptor tetramers is regulated by RNA editing at the $\mathrm{Q} / \mathrm{R}$ site in the pore loop [46]. Relevant to this is a growing realization that the pore structure of iGluRs is not 4-fold symmetric [47॰ ].

\section{Conclusions}

Structural work on iGluRs has undergone an explosive burst of activity such that it is now possible to explain at the atomic level the ligand binding properties of key individual subtypes. It is anticipated that similar work on the NR2 glutamate binding NMDA receptor subunits as well as other less well characterized iGluRs will complete this picture in the near future. This should provide the groundwork for enormous advances in medicinal chemistry. As illustrated in Figure 2 the ligand binding cores account for a mere $25 \%$ of the mass of an AMPA receptor subunit, and substantially less in the case of NMDA receptor NR2 subunits which have 50-70 kDa cytoplasmic C terminal domains. Structural information is almost entirely lacking for the remaining $75 \%$ of an iGluR subunit and as a result the molecular mechanisms underlying ion permeation, gating, assembly, trafficking, and activity dependent modulation are much less well understood. 
This is unlikely to change until the technical challenges of working with eukaryotic membrane proteins are solved.

References and recommended reading.

1. Armstrong N, Sun Y, Chen GQ, Gouaux E: Structure of a glutamate-receptor ligand-binding core in complex with kainate. Nature 1998, 395:913-917.

- 2. Mayer ML: Crystal Structures of the GluR5 and GluR6 Ligand Binding Cores: Molecular Mechanisms Underlying Kainate Receptor Selectivity. Neuron 2005:539-552.

A series of six high resolution structures reveal the mechanisms underlying kainate receptor subtype selectivity, how AMPA and kainate receptors differ in their ligand binding properties, and interdomain contacts unique to kainate receptors.

- 3. Furukawa H, Gouaux E: Mechanisms of activation, Inhibition and Specificity: Crystal Structures of NR1 Ligand-Binding Core. Embo J 2003, 22:1-13.

High resolution structures of the NR1 subunit glycine binding site reveals how NMDA receptors discriminate between glutamate and glycine, and structures of antagonist and partial agonist complexes.

4. Naur P, Vestergaard B, Skov LK, Egebjerg J, Gajhede M, Kastrup JS: Crystal structure of the kainate receptor GluR5 ligand-binding core in complex with (S)-glutamate. FEBS Lett 2005, 579:1154-1160.

5. Nanao MH, Green T, Stern-Bach Y, Heinemann SF, Choe S: Structure of the kainate receptor subunit GluR6 agonist-binding domain complexed with domoic acid. Proc Natl Acad Sci U S A 2005:1708-1713.

6. Armstrong N, Gouaux E: Mechanisms for activation and antagonism of an AMPAsensitive glutamate receptor: Crystal structures of the GluR2 ligand binding core. Neuron 2000, 28:165-181.

7. Lunn ML, Hogner A, Stensbol TB, Gouaux E, Egebjerg J, Kastrup JS: Threedimensional structure of the ligand-binding core of GluR2 in complex with the agonist (S)-ATPA: implications for receptor subunit selectivity. $J$ Med Chem 2003, 46:872-875.

8. Valentine ER, Palmer AGI: Microsecond-to-millisecond conformational dynamics demarcate the GluR2 glutamate receptor bound to agonists glutamate, quisqualate, and AMPA. Biochemistry 2005, 44:3410-3417.

9. Zeng L, Chen CH, Muller M, Zhou MM: Structure-based rational design of chemical ligands for AMPA-subtype glutamate receptors. $J$ Mol Neurosci 2003, 20:345-348.

10. Hogner A, Greenwood JR, Liljefors T, Lunn ML, Egebjerg J, Larsen IK, Gouaux E, Kastrup JS: Competitive antagonism of AMPA receptors by ligands of different classes: crystal structure of ATPO bound to the GluR2 ligandbinding core, in comparison with DNQX. J Med Chem 2003, 46:214-221. 
11. More JC, Nistico R, Dolman NP, Clarke VR, Alt AJ, Ogden AM, Buelens FP, Troop HM, Kelland EE, Pilato F, et al.: Characterisation of UBP296: a novel, potent and selective kainate receptor antagonist. Neuropharmacology 2004, 47:46-64.

- 12. Inanobe A, Furukawa H, Gouaux E: Mechanism of partial agonist action at the NR1 subunit of NMDA receptors. Neuron 2005.

This study reveals how localized structural changes accommodate the binding of glycine site partial agonists without altering domain closure, distinct from the mechanism for partial agonist action at wild type AMPA receptors.

13. Chambers JJ, Gouda H, Young DM, Kuntz ID, England PM: Photochemically knocking out glutamate receptors in vivo. $J$ Am Chem Soc 2004, 126:1388613887.

14. Foucaud B, Laube B, Schemm R, Kreimeyer A, Goeldner M, Betz H: Structural model of the N-methyl-D-aspartate receptor glycine site probed by sitedirected chemical coupling. J Biol Chem 2003, 278:24011-24017.

15. Laube B, Schemm R, Betz H: Molecular determinants of ligand discrimination in the glutamate-binding pocket of the NMDA receptor. Neuropharmacology 2004, 47:994-1007.

16. Chen PE, Geballe MT, Stansfeld PJ, Johnston AR, Yuan H, Jacob AL, Snyder JP, Traynelis SF, Wyllie DJ: Structural features of the glutamate binding site in recombinant NR1/NR2A N-methyl-D-aspartate receptors determined by sitedirected mutagenesis and molecular modeling. Mol Pharmacol 2005, 67: 14701484.

-๑ 17. Jin R, Banke TG, Mayer ML, Traynelis SF, Gouaux E: Structural basis for partial agonist action at ionotropic glutamate receptors. Nature Neurosci 2003, 6:803-810.

This detailed mechanistic analysis combines crystallographic studies with whole cell recording to reveal how graded increases in the size of an AMPA receptor agonist progressively reduce domain closure to produce partial agonist activity. Single channel channel recording reveals that partial agonist activity alters the relative occupancy of discrete set of sub conductance states.

- 18. Armstrong N, Mayer M, Gouaux E: Tuning activation of the AMPA-sensitive GluR2 ion channel by genetic adjustment of agonist-induced conformational changes. Proc Natl Acad Sci U S A 2003, 100:5736-5741.

Mutation of a binding site residue that in wild type receptors 'collides' with kainate is shown to increase both domain closure and efficacy for kainate and to destabilize the fully closed conformation for AMPA.

19. Hogner A, Kastrup J, Jin R, Liljefors T, Mayer M, Egebjerg J, Larsen I, Gouaux E: Structural Basis for AMPA Receptor Activation and Ligand Selectivity: Crystal Structures of Five Agonist Complexes with the GluR2 Ligandbinding Core. J Mol Biol 2002, 322:93.

20. Frandsen A, Pickering DS, Vestergaard B, Kasper C, Nielsen BB, Greenwood JR, Campiani G, Fattorusso C, Gajhede M, Schousboe A, et al.: Tyr702 Is an 
Important Determinant of Agonist Binding and Domain Closure of the Ligand-Binding Core of GluR2. Mol Pharmacol 2005, 67:703-713.

21. Madden DR, Cheng Q, Thiran S, Rajan S, Rigo F, Keinanen K, Reinelt S, Zimmermann H, Jayaraman V: Stereochemistry of glutamate receptor agonist efficacy: engineering a dual-specificity AMPA/kainate receptor. Biochemistry 2004, 43:15838-15844.

22. Fleck MW, Cornell E, Mah SJ: Amino-acid residues involved in glutamate receptor 6 kainate receptor gating and desensitization. J Neurosci 2003, 23:1219-1227.

23. Tichelaar W, Safferling M, Keinanen K, Stark H, Madden DR: The Threedimensional Structure of an Ionotropic Glutamate Receptor Reveals a Dimer-of-dimers Assembly. J Mol Biol 2004, 344:435-442.

24. Sun Y, Olson R, Horning M, Armstrong N, Mayer M, Gouaux E: Mechanism of glutamate receptor desensitization. Nature 2002, 417:245-253

- 25. Horning MS, Mayer ML: Regulation of AMPA receptor gating by ligand binding core dimers. Neuron 2004, 41:379-388.

An extensive functional analysis of AMPA receptor ligand binding core dimer contacts reveals how subtle changes in amino acid chemistry profoundly influence gating kinetics.

- 26. Nakagawa T, Cheng Y, Ramm E, Sheng M, Walz T: Structure and different conformational states of native AMPA receptor complexes. Nature 2005, 433:545-549.

Single particle electron microscopy and mass spectrometry reveal conformational changes linked with desensitization and that the membrane embedded segment of native AMPA receptors forms a complex with TARPs.

- 27. Yelshansky MV, Sobolevsky AI, Jatzke C, Wollmuth LP: Block of AMPA receptor desensitization by a point mutation outside the ligand-binding domain. J Neurosci 2004, 24:4728-4736.

The identification of amino acids outside the ligand binding core which regulate desensitization has the potential to open a new door leading to a better understanding of iGluR gating mechanisms.

28. Quirk JC, Nisenbaum ES: Multiple molecular determinants for allosteric modulation of alternatively spliced AMPA receptors. J Neurosci 2003, 23:10953-10962.

29. Leever JD, Clark S, Weeks AM, Partin KM: Identification of a site in GluR1 and GluR2 that is important for modulation of deactivation and desensitization. Mol Pharmacol 2003, 64:5-10.

30. Kim E, Sheng M: PDZ domain proteins of synapses. Nat Rev Neurosci 2004, 5:771-781.

31. Bowie D, Garcia EP, Marshall J, Traynelis SF, Lange GD: Allosteric regulation and spatial distribution of kainate receptors bound to ancillary proteins. $J$ Physiol 2003, 547:373-385. 
๑ 32. Tomita S, Hillel A, Sekiguchi M, Zhang W, Wada K, Howe JR, Nicoll RA, Bredt DS: Stargazin modulates AMPA receptor gating and trafficking by distinct domains. Nature 2005, in press.

The demonstration that the $\gamma-2$ member of the TARP family modulates AMPA receptor gating, single channel conductance, and synaptic function has profound implications for numerous aspects of iGluR biology and will undoubtedly stimulate work in many groups. An elegant molecular dissection suggests regions of the TARP molecule play different roles in trafficking and modulation.

33. Tomita S, Chen L, Kawasaki Y, Petralia RS, Wenthold RJ, Nicoll RA, Bredt DS:

Functional studies and distribution define a family of transmembrane AMPA receptor regulatory proteins. J Cell Biol 2003, 161:805-816.

34. Vandenberghe $\mathrm{W}$, Nicoll RA, Bredt DS: Stargazin is an AMPA receptor auxiliary subunit. Proc Natl Acad Sci U S A 2005, 102:485-490.

35. Popescu G, Auerbach A: Modal gating of NMDA receptors and the shape of their synaptic response. Nat Neurosci 2003, 6:476-483.

36. Banke TG, Dravid SM, Traynelis SF: Protons trap NR1/NR2B NMDA receptors in a nonconducting state. J Neurosci 2005, 25:42-51.

37. Popescu G, Robert A, Howe JR, Auerbach A: Reaction mechanism determines NMDA receptor response to repetitive stimulation. Nature 2004, 430:790-793.

38. Hatton CJ, Paoletti P: Modulation of triheteromeric NMDA receptors by Nterminal domain ligands. Neuron 2005, in press.

39. Rachline J, Perin-Dureau F, Le Goff A, Neyton J, Paoletti P: The micromolar zincbinding domain on the NMDA receptor subunit NR2B. J Neurosci 2005, 25:308-317.

40. Mott DD, Washburn MS, Zhang S, Dingledine RJ: Subunit-dependent modulation of kainate receptors by extracellular protons and polyamines. $J$ Neurosci 2003, 23:1179-1188.

41. Turecek R, Vlcek K, Petrovic M, Horak M, Vlachova V, Vyklicky L, Jr.: Intracellular spermine decreases open probability of $\mathrm{N}$-methyl-D-aspartate receptor channels. Neuroscience 2004, 125:879-887.

42. Yan S, Sanders JM, Xu J, Zhu Y, Contractor A, Swanson GT: A C-terminal determinant of GluR6 kainate receptor trafficking. J Neurosci 2004, 24:679691.

43. Schorge S, Colquhoun D: Studies of NMDA receptor function and stoichiometry with truncated and tandem subunits. J Neurosci 2003, 23:1151-1158.

44. Valluru L, Xu J, Zhu Y, Yan S, Contractor A, Swanson GT: Ligand binding is a critical requirement for plasma membrane expression of heteromeric kainate receptors. J Biol Chem 2005, 280:6085-6093.

45. Mah SJ, Cornell E, Mitchell NA, Fleck MW: Glutamate receptor trafficking: Endoplasmic reticulum quality control involves ligand binding and receptor function. Journal of Neuroscience 2005, 25:2215-2225. 
46. Greger IH, Khatri L, Kong X, Ziff EB: AMPA receptor tetramerization is mediated by Q/R editing. Neuron 2003, 40:763-774.

Elegant biochemical studies reveal how RNA editing of the GluR2 AMPA receptor subunit affects assembly prior to transport to the plasma membrane

47. Sobolevsky AI, Yelshansky MV, Wollmuth LP: The outer pore of the glutamate receptor channel has 2-fold rotational symmetry. Neuron 2004, 41:367-378.

- 48. Priel A, Kolleker A, Ayalon G, Gillor M, Osten P, Stern-Bach Y. Stargazin reduces desensitization and slows deactivation of the AMPA-type glutamate

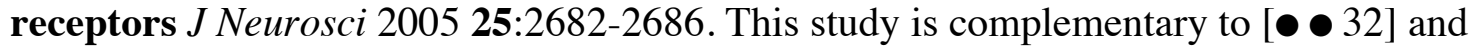
focuses on the novel result that TARPs modulate AMPA receptor gating kinetics. 
A

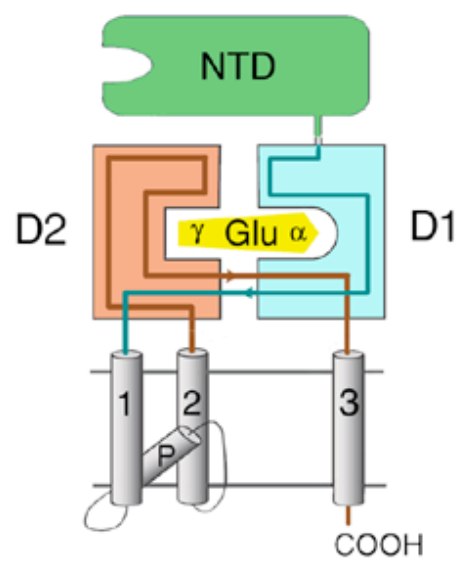

B

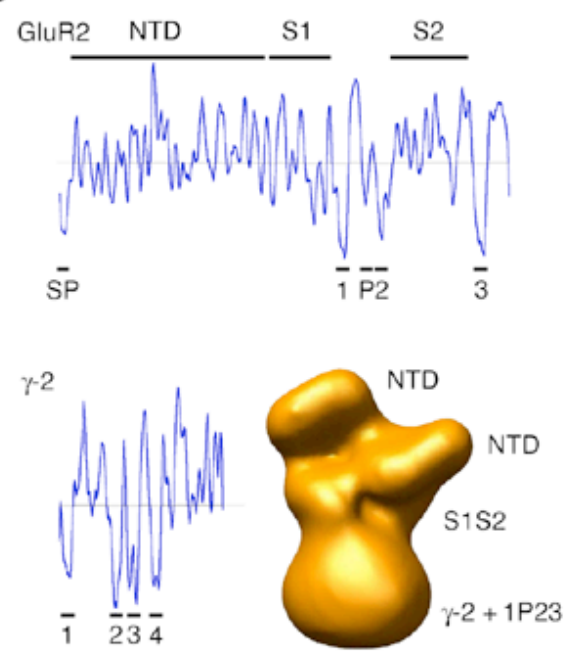

Figure 1 Domain structure in glutamate receptor ion channels. (A) Each subunit consists of a bilobed amino terminal domain (NTD), the two domain ligand binding core (D1 D2), an ion channel with 3 membrane spanning segments (1-3) and a pore loop (P), and a cytoplasmic domain of variable length. (B) Low resolution structures of iGluRs and accessory subunits illustrated by hydropathy plots for GluR2 (top) and the $\gamma-2$ subunit of the TARP family (lower left), and by a single particle image of an AMPA receptor complex with TARPs [26]. 

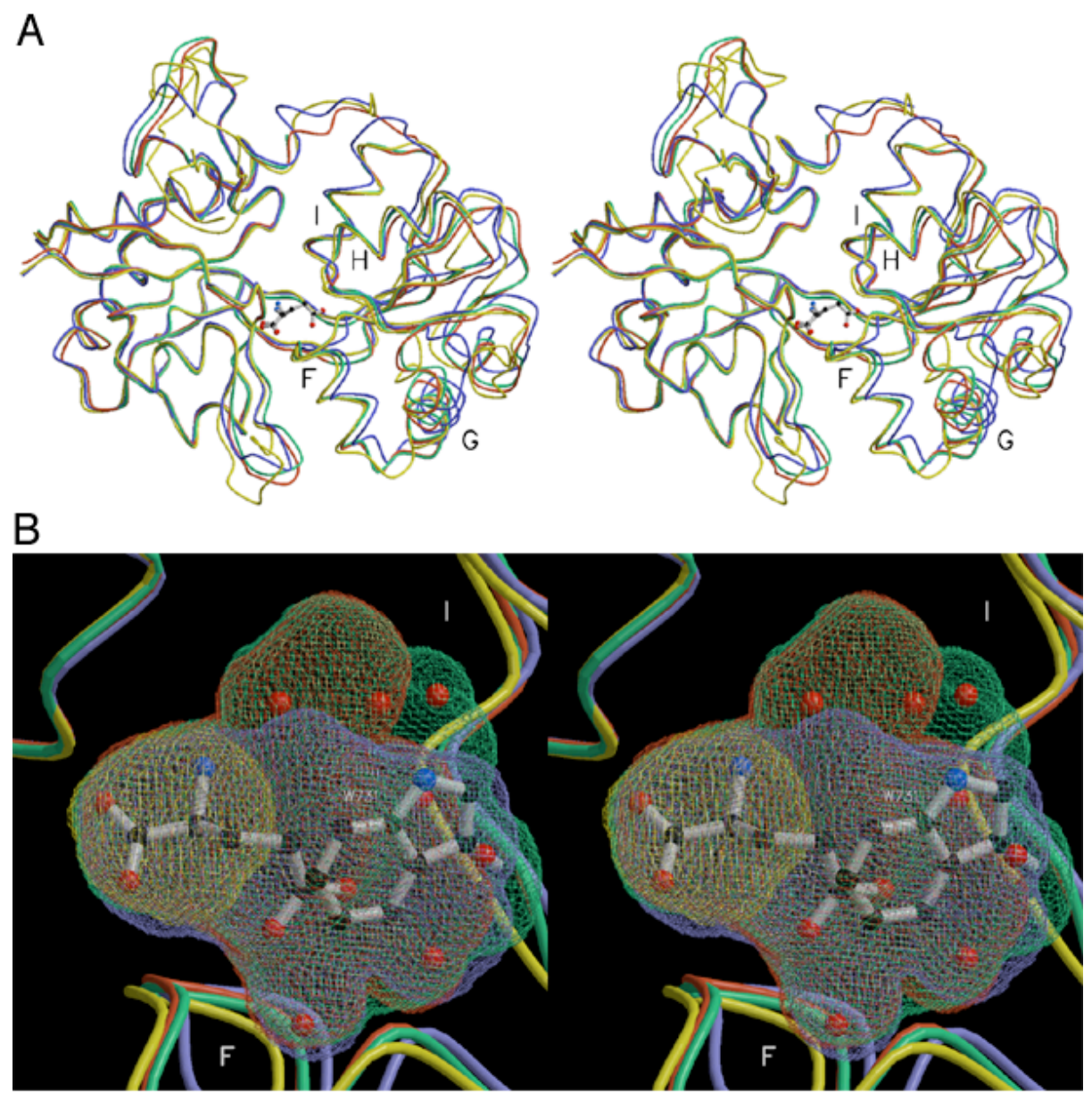

Figure 2 Crystal structures of the agonist bound complexes for the ligand binding domains of iGluRs. (A) Stereoview of NR1 (yellow). GluR2 (blue), GluR5 (green) and GluR6 (red) superimposed using domain 1 coordinates $[2,3,6]$; the positions of helices $\mathrm{F}$, $\mathrm{G} \mathrm{H}$ and $\mathrm{I}$ in domain 2 are indicated by labels. Ball and stick representations show the glycine ligand from NR1 and the glutamate ligand from GluR6 for which the $\alpha$-carbon functional groups almost completely overlap. (B) Stereoview of solvent accessible surfaces of the ligand binding pocket interiors; the surfaces and coils are colored using the same scheme as used above to illustrate secondary structure surrounding the binding site; helices $\mathrm{F}$ and $\mathrm{I}$ in domain 2 are labeled for orientation. Ball and stick representations show the glutamate ligand and six water molecules trapped in the GluR5 ligand binding pocket and the W731 side chain of NR1. All of the water molecules lie outside the NR1 cavity, which is just big enough to accommodate glycine; the upper 3 water molecules in the GluR5 structure also lie outside the envelope of the GluR2 cavity; the top right water molecule in addition lies outside the envelope of the GluR6 cavity. W731 projects into the cavity of the AMPA and kainate receptors preventing the binding of glutamate to NR1. 